\title{
DEMYSTIFYING DIGITAL X
}

\author{
Cox, Chris (1); \\ Gopsill, James (1,2); \\ Hicks, Ben (1)
}

1: Design Manufacturing Futures Lab, School of Civil, Aerospace and Mechanical Engineering,University of Bristol;

2: Centre for Modelling and Simulation, Bristol, UK

\section{ABSTRACT}

The rapid pace of development in Digital Engineering has led to an explosion of ideas and new practice in how it can support Engineering Design and Manufacture. You may have heard of the terms Digital Transformation, Digital Twin, Digital Thread, Digital Tapestry and Digital Footprint amongst many other forms of "Digital X" but how have these come about and how do they come together to provide the landscape of what Digitalisation has to offer?

In this paper, we analyse the emergence, definition, use and co-occurrence of "Digital X" terminology from an academic dataset of 19,627 papers curated from Scopus. The results reveal that these terms are being used without being fully contextualised in terms of a hierarchy or equivalent to effectively articulate the Digital landscape.

Through this analysis, an emerging "Digital X" framework is proposed, with evidence given to support suggested links, and knowledge gaps highlighted for further investigation. Once this framework is complete, a rich lexicon describing the Digital Landscape will pave the way for the future in Digital Engineering.

Keywords: Digital / Digitised engineering value chains, Ontologies, Communication, Co-word analysis, Digital transformation

\section{Contact:}

Cox, Christopher Michael Jason

University of Bristol

Mechanical Engineering

United Kingdom

christopher.cox@bristol.ac.uk 


\section{INTRODUCTION}

Engineering practice is radically and rapidly transforming by digital technology that either complements or supersedes physical practice (e.g., paper-based reporting). With new technology being developed every day, it has become difficult to keep track of every new innovation and methodology, let alone be able to communicate and discuss it through language. A language is emerging that is highly-transient and chaotic with numerous terms being developed and used to inform and frame Digital Engineering. Terms such as the 'Digital X' family; for example, Digital Transformation, Digital Economy Digital Twin and Digital Thread. And there remains much confusion in what these terms mean and how they fit together to provide an overall framework of what is referred to herein as Digital Engineering.

To take full advantage of digital technology, an understanding of what these terms mean and how they relate to each other is required. This is a prerequisite for industry to understand their current maturity and determine how, where and when to deploy digital technologies in order to continue or start their Digital Transformation.

To demystify Digital ' $X$ ', this paper's contribution is an analysis of academic literature to investigate the emergence of "Digital X" terminology and how it relates to and defines Digital Engineering. This is achieved through the analysis of term use and co-occurrence. Resulting from this, the paper proposes how these terms are related to each other, the continuing tensions between Digital ' $X$ ' terminology and any gaps that require further research.

The paper starts by describing the review methodology that has taken academic literature as the input (Section 2). The results are then presented (Section 3) along with a discussion of the impact of Digital ' $X$ ' terminology and its influence on the definition and framing of Digital Engineering. These results are then used as the evidence for a Digital ' $X$ ' framework that represents the current set of terms and interrelationships that collectively define Digital ' $X$ ' (Section 4). This is followed by a comparison of the elicited academic definitions to those proposed by industry and, in particular, the major consulting companies leading the digital transformation. The implications of the findings on Engineering Design research and practice are elicited alongside further work to gain a deeper understanding of this new and evolving lexicon (Section 4.7). The paper then concludes with the key findings of the study (Section 5).

\section{REVIEW METHODOLOGY}

The review of academic literature applied a four step descriptive research methodology with a mix of qualitative and quantitative analysis. The process is outlined in Figure 1 and returned a substantial dataset. Thus, it would be impractical to conduct a manual analysis of each document, therefore an automated scheme was devised. Analysis of the usage of different terms was important to see which terms dominate, and which terms are less commonly used. Gainsburg et al. (2010) and Yang et al. (2012) highlight the importance of terminology in engineering and how diverse language can lead to misunderstanding and confusion in the design process, whilst consistent re-use of terminology is an indication of shared understanding with designers working towards to common agreed goal. Temporal analysis was required so that the variation of term frequency over time could be assessed. This facilitates the identification of leading and following terms, as well as framing the time-scale for the rise of Digital "X". Gainsburg et al. (2010) showed that knowing the origins of the knowledge and terminology used is vital in engineering, supporting the use of temporal analysis in this paper. Co-occurrence analysis is a powerful tool for the analysis of interrelationships between a group of terms, as shown by Callon, Courtial, and Laville (1991), Callon, Courtial, Turner, et al. (1983), and Yang et al. (2012). This tool would be vital to establish a framework within the "Digital X" family.

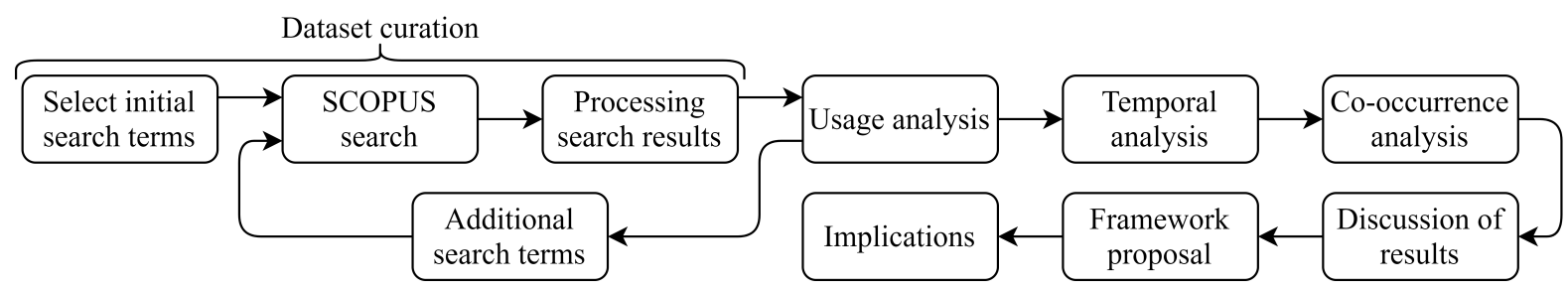

Figure 1. Methodology diagram 


\subsection{Dataset Curation}

To start, an initial set of documents were found using SCOPUS ${ }^{1}$, with search terms in the format of "Digital X". This format was selected as it had been identified as a common theme for many recent phrases that aim to describe an aspect of the Digital Engineering landscape. SCOPUS was selected as it provides a comprehensive, general academic publication database and evaluation has shown it to provide $20 \%$ more coverage and incorporates a wider range of journals than competing services, such as the Web of Science (Aghaei Chadegani et al., 2013).

Initial search terms were selected based on the interests of our collaborators investigating the "Digital Thread" on the Digital Engineering and Technology \& Innovation (DETI) project. By investigating the history of this term we identified its origins linked to a holistic method for design and manufacture developed by NASA and Lockheed Martin called the "Digital Tapestry" (Gaska et al., 2015). Other closely related terms fitting the format "Digital X" were found to be "Digital Twin" and "Digital Transformation".

Further investigation of searches using Digital ' $\mathrm{X}$ ' n-gram analysis revealed a number of other popular "Digital X" terms. Digital ' $\mathrm{X}$ ' terms with a frequency of $100 \leq f \leq 10,000$ were used as additional search terms to produce a final document dataset from SCOPUS.

The $\leq 11,000$ clause was used to remove context-independent terminology. One example was the term "Digital Storage", which returned over 100,000 results, and would likely only be loosely related to the terms being investigated. Variations on these terms were also included in the search, accounting for plurality and grammatical application, i.e., manufacturing/manufacture.

These steps resulted in the following search terms for the review.

- Digital Twin

- Digital Thread

- Digital Transformation
- Digital Tapestry

- Digital Technology

- Digital Engineering
- Digital Economy

- Digital Manufacturing

- Digital Footprint

Searches with these terms resulted in 20,496 documents being returned. Subsequent post-processing of the papers, included the automated removal of duplicates and manual removal of early documents unrelated to the topics being assessed in this review (discussed further in Section 3). This resulted in a final dataset of 19,627 documents. The information used from these documents included the abstract, keywords and date published.

\subsubsection{Dataset Validation}

Having identified nine key Digital ' $\mathrm{X}$ ' terms, it was important to verify that these were unique terms with a distinct meaning, and not simply common word pairs. An example of this is discussed in more detail in Section 3.1 and is the use of the phrase "digital thread" in a paper documenting "digital thread counters" by GmbH and Co. (1996). The appearance of the words "digital thread" in this case does not refer to a unique term but is a part of a different term entirely, referring to a digital tool for counting threads.

To verify that the nine identified Digital ' $X$ ' terms were meaningful to Digital Engineering, the top cited 10 papers for each term on the SCOPUS database were examined to check for a distinct meaning.

\subsubsection{Dataset Preparation}

Analysis of the dataset required the text to be standardised in order to collect key terms together regardless of formatting. To achieve this, the text in the abstract was processed as shown in Figure 2.

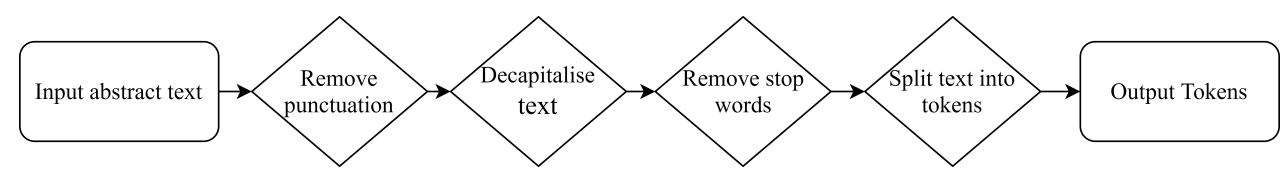

Figure 2. Preparation process for abstract text

\footnotetext{
${ }^{1}$ An abstract and citation database by Elsevier.
} 


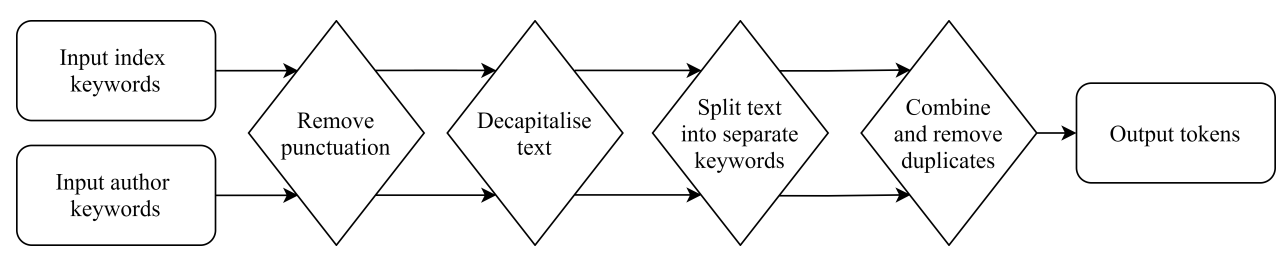

Figure 3. Preparation process for keyword lists

For each abstract, the process removed punctuation, decapitalised the text and removed stopwords. The list of stopwords used was taken from the Natural Language Toolkit (NLTK) Python package (Loper and Bird, 2002). The remaining text was then split on whitespace to form a token set for the abstract. The preparation for the keywords differed as stopwords did not need to be removed, and further processing was needed to filter between the two sets - index and author - of keywords provided by SCOPUS. It was decided that both sets of keywords were equally important as the authors will have a more intimate knowledge of the content, but the SCOPUS indexing algorithm is better suited to categorise the document using common keywords. As there was overlap between these two sets, for each document the keywords were combined and duplicates removed. The process is summarised in Figure 3.

\subsection{Usage Analysis}

A word cloud was selected to display how often terms were used relative to each other. The word cloud displays words on a page with their relative size representing how many documents each phrase or keyword appeared in. The methods is commonly applied document reviews (DePaolo and Wilkinson, 2014).

A word cloud was created for the most common key words, and the most common bigrams and trigrams found in the abstracts. Prior work has shown that bigrams and trigrams are more suitable in identifying engineering related terminology (Gopsill et al., 2020). The results of the n-gram analysis were further post-processed to remove publishing information and common academic phrases such as "this paper" and "rights reserved".

\subsection{Temporal Analysis}

Temporal analysis was performed to investigate the emergence and use (occurrence) of Digital ' $\mathrm{X}$ ' terminology. This was achieved by scanning the dataset for each keyword and producing a list of documents that featured it. Ordering these documents by their publication date enabled a graph to be produced to show the temporal frequency of use of key terms.

Some documents in the dataset were shown as being published in 2021, these have been included in the 2020 date as they were in early access at the time of writing. It is also worth noting that there is a higher degree of uncertainty in the 2020 results as not all documents published this year will have been added to SCOPUS at the time of writing.

\subsection{Co-Occurrence Analysis}

To calculate the co-occurrence between Digital ' $\mathrm{X}$ ' terms, $t$, in the term set, $T, t \in T$, the overlap in documents, $d$, in the document set, $D$, containing each term, $t \in d \in D$, either in the abstract or keywords, was tallied for each term pair. This was then normalised to see the relative impact of each term upon one another, $c$, (Equation (1)).

$$
c_{i j}=\frac{\sum_{k=1}^{n}\left\{t_{i}, t_{j}\right\} \in d_{k}}{\sum_{k=1}^{n} t_{i} \in d_{k}}
$$

Where $\sum_{k=1}^{n}\left\{t_{i}, t_{j}\right\} \in d_{k}$ is the sum of documents that feature both terms, with $\left\{t_{i}, t_{j}\right\} \in d_{i}$ being either 1 if $t_{i}$ and $t_{j}$ are both in document $d_{k}$, or 0 otherwise. $\sum_{k=1}^{n} t_{i} \in d_{k}$ is the sum of documents with term $t_{i}$ in them. Again, returning 1 if $t_{i}$ is in document $d_{k}$, or 0 otherwise. The normalisation was carried out in both directions, to determine the impact of term $i$ on term $j$ and vice versa, enabling us to analyse the directionality of the relationship. 


\section{RESULTS}

This section details the results of the review. It starts by providing a summary of the curated dataset, followed by the results of the usage, temporal and co-occurrence analysis in Sections 3.2, 3.3 and 3.4 respectively.

\subsection{Dataset Summary}

Table 1 summarises the dataset in terms of documents found for each search term, the first and last publication dates where the term appeared, and how many documents were published pre-2012. Details of the methodology's implementation via code is provided in the accompanying data repository, which can be found at data.bris.ac.uk.

Table 1. A summary of the documents analysed in this paper. Note: Documents before 2012 for "digital technology" were removed from the dataset and are not included in the analysis

\begin{tabular}{l|rrr|r}
\hline & \multicolumn{3}{|c|}{ Number of Documents: } & \\
Search Term & Found & Removed & Pre-2012 & Start - End Dates \\
\hline Digital Transformation & 4,765 & 56 & 140 & $1999-2021$ \\
Digital Twin & 2,658 & 2 & 7 & $1994-2021$ \\
Digital Thread & 154 & 2 & 4 & $2009-2020$ \\
Digital Tapestry & 9 & 4 & 2 & $2014-2018$ \\
Digital Economy & 3,864 & 0 & 580 & $1984-2021$ \\
Digital Engineering & 327 & 0 & 153 & $1969-2021$ \\
Digital Footprint & 565 & 2 & 55 & $2007-2021$ \\
Digital Manufacture & 1,523 & 35 & 447 & $1999-2021$ \\
$\begin{array}{l}\text { Digital Technology (Limited to } \\
\text { Engineering, Maths, Computer Sci- } \\
\text { ence, and post 2012) }\end{array}$ & 10,412 & 3,753 & 3,753 & $1963-2021$ \\
\hline
\end{tabular}

It is surprising to see some of these search terms having much earlier first publication dates than one might expect. These were investigated and found to be due to the use of the word "digital" in other applications. For example, a document on a "Digital thread counter" by GmbH and Co. (1996) used in automated weaving machines was published in 1996. There are also cases of documents that use the same term but with different intent. For example a number of documents in the early 1970's by Kinberg and Landeck (1970) and Stuehler (1970) discuss the manufacture of IBM's digital computers. This "digital manufacture" refers to the manufacture of digital components, rather than the digitisation of the manufacturing process itself which the term has come to mean now.

To provide an accurate point at which each term was first used in the context of Digital Engineering, for each search term, the earliest documents were checked for relevance and filtered. This process was continued until the earliest relevant document was found. The number of documents removed for each search term is recorded in Table 1 . There is likely to still be some uncertainty remaining in the dataset, but as the rate of publication of documents within the dataset increases in the latter years, it is argued that this will have had a negligible impact on the subsequent analysis.

The dataset also featured some missing data. Out of the 19,627 documents, 688 (3.5\%) were missing abstracts and 2,129 (11\%) were missing both index and author keywords. These documents have been included in the analysis nonetheless as they still contribute to the temporal analysis. The relatively low percentage also provides us with confidence that the trends observed from the analysis are not likely to be swayed by the future addition of the missing data.

\subsubsection{Dataset Verification and Definitions}

Using the methodology outlined in Section 2.1.1, the definitions for the chosen Digital ' $\mathrm{X}$ ' terms were derived and are summarised in Table 2.

Unfortunately, some of the terms that were chosen are so widely used that they are often used without consideration of their meaning. These high level, abstract terms were "Digital Technology", "Digital Transformation", "Digital Manufacturing" and "Digital Economy". It suggests that the meanings are self-evident but it is interesting to see that there is little to no research that has tested this assumption. 
Table 2. Digital ' $X$ ' academic definitions.

\begin{tabular}{|c|c|c|}
\hline \# & Definition & Ref. \\
\hline \multicolumn{3}{|c|}{ Digital Transformation } \\
\hline 1 & The exploitation and integration of digital technologies [within a company]. & (Matt et al., 2015) \\
\hline 2 & Integrating and exploiting new digital technologies. & (Hess et al., 2016) \\
\hline \multicolumn{3}{|c|}{ Digital Twin } \\
\hline 1 & $\begin{array}{l}\text { An integrated multi-physics, multi-scale, and probabilistic simulation of a complex } \\
\text { product [which] uses the best available physical models, sensor updates, etc., to mirror } \\
\text { the life of its corresponding twin. }\end{array}$ & (Tao et al., 2018) \\
\hline 2 & $\begin{array}{l}\text { Very realistic models of the current state of the process and their own behaviour in } \\
\text { interaction with their environment in the real world. }\end{array}$ & (Rosen et al., 2015) \\
\hline \multicolumn{3}{|c|}{ Digital Thread } \\
\hline 1 & $\begin{array}{l}\text { Allows for rapid communication, iteration, and sharing of a design model and its } \\
\text { corresponding physical representation. }\end{array}$ & (Sturm et al., 2017) \\
\hline 2 & A common software toolchain within a project. & (Steuben et al., 2016) \\
\hline 3 & $\begin{array}{l}\text { The information and information path that is gathered and stored when manufacturing } \\
\text { a single part. }\end{array}$ & (Kim et al., 2015) \\
\hline 4 & The flow of information along the product lifecycle and across the supply network. & (Feeney et al., 2015) \\
\hline 5 & $\begin{array}{l}\text { Data is captured throughout the product lifecycle and analysed for opportunities to drive } \\
\text { down tooling costs and lead times and improve efficiencies and innovation. }\end{array}$ & (Mies et al., 2016) \\
\hline \multicolumn{3}{|c|}{ Digital Tapestry } \\
\hline 1 & $\begin{array}{l}\text { [A method of] seamlessly connecting computer design tools, modelling and simulation, } \\
\text { intelligent machines and sensors, additive manufacturing, manufacturing methods, and } \\
\text { post-delivery services to shorten the time and cost between idea generation and first } \\
\text { successful product-in-hand. }\end{array}$ & (Bullen, 2014) \\
\hline \multicolumn{3}{|c|}{ Digital Footprint } \\
\hline 1 & The collective, ongoing record of one's Web activity. & (O’Keeffe et al., 2011) \\
\hline 2 & User-generated electronic trails. & (Girardin et al., 2008) \\
\hline
\end{tabular}

The terms that have definitions for them may suggest that it is important to consider the contextual nature of these terms (i.e., the application of the term in the Digital Engineering context) and that a context/situated meaning is essential for understanding their utility in the Digital Landscape. We also observe some terms, such as Digital Transformation and Digital Footprint, having few, and in some cases, repeated definitions by academics which shows a degree of convergence. However others, such as Digital Thread, have numerous definitions revealing a lack of agreement in the field as to what the term represents, frustrating its localisation in the Digital Landscapes. While a detailed analysis of definitions is out of the scope of this paper, Table 2 confirms that these are all terms that are describing features of Digital Engineering.

\subsection{Word Clouds - Usage Analysis}

The word clouds of the most common n-grams from the document abstracts, and most common keywords are shown in Figure $4 \mathrm{a}$ and $4 \mathrm{~b}$, respectively. The number of terms in the word cloud is determined by the available space rather than a fixed number. In order to provide context to these, a list of top terms in each word cloud and the number of documents they appear in is shown in Table 3.

The number of documents a term occurs in is used as the metric, instead of the total number of occurrences, as the total occurrences will be a function of personal writing preference, whereas the number of documents is more dependant on the document topic itself.

As might be expected, the original search terms are amongst the most popular terms found across the dataset. Beyond this however, there is some insight into which other terms not of the form Digital ' $\mathrm{X}$ ' are popular within this space. For example, 'Industry 4.0' appears frequently, as might be expected seeing this term is used to describe the digital transformation of the business and engineering world. Other popular terms include Internet of Things (IoT), Big Data, Artificial Intelligence and more. These terms are related to the Digital ' $X$ ' family discussed in this paper and will require further investigation. 


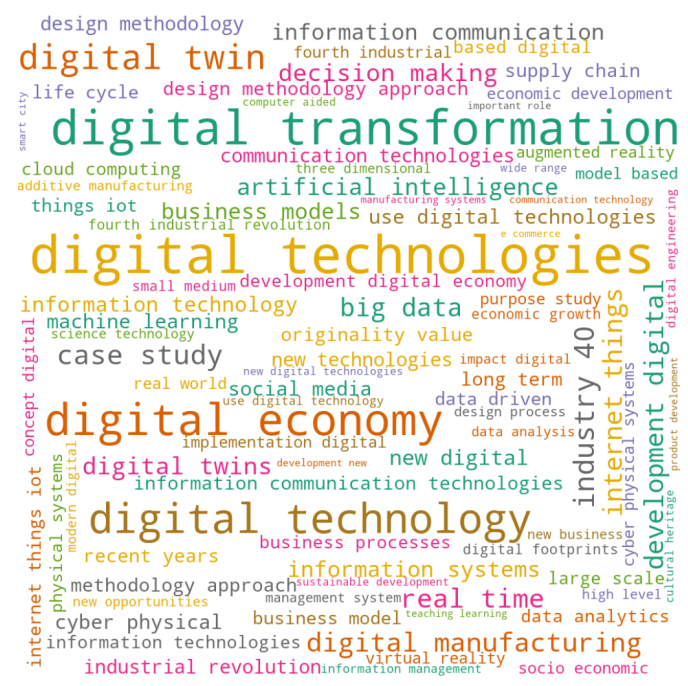

(a) Word cloud of most common bi-grams and tri-grams

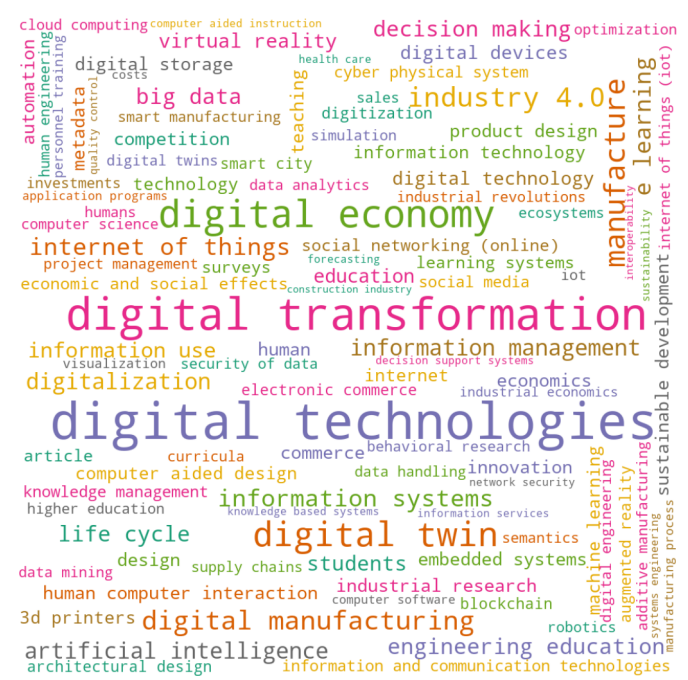

(b) Word cloud of most common keywords

Figure 4. Word clouds of most common terms across the dataset

Table 3. Top results from the $n$-gram and keyword usage analysis

\begin{tabular}{lclc}
\hline Top n-grams & Occurrences & Top keywords & Occurrences \\
\hline digital technologies & 4342 & digital technologies & 3878 \\
digital transformation & 3515 & digital transformation & 2806 \\
digital technology & 2893 & digital economy & 1764 \\
digital economy & 2849 & digital twin & 1603 \\
digital twin & 1783 & manufacture & 1157 \\
case study & 1102 & industry 4.0 & 957 \\
industry 40 & 1088 & digital manufacturing & 926 \\
real time & 1027 & information systems & 775 \\
digital manufacturing & 988 & internet of things & 763 \\
internet (of) things & 930 & e learning & 733 \\
\hline
\end{tabular}

\subsection{Digital ' $X$ ' Over Time - Temporal Analysis}

The temporal analysis results are shown in Figure 5. This shows the number of documents featuring each key term published per year between 2012 and 2020. This time frame was selected as the prior data did not provide much more insight, as all terms had negligible occurrences, or a slow linear increase.

The early prominence of Digital Technology is logical, the technology must exist before a lexicon can be framed around it. There is then an observed lag of approximately five years before we see a ramp in other Digital ' $X$ ' terminology, likely as there was insufficient technology before this to consider the need for a specific framing of the landscape.

The order of prominence across Digital ' $\mathrm{X}$ ' remains constant throughout the years with Digital Technology followed by Digital Transformation, Digital Economy, and then Digital Twin. Of these, the three former terms start rising in the same year, implying a strong inter-relationship. This is as expected as Digital Technology is needed to undergo a digital transformation, and you must transform digitally before you can start contributing to the Digital Economy. This is a cyclical dependency as the Digital Economy will promote more Digital Technology and Digital Transformation, leading to an almost symbiotic relationship.

The Digital Twin starts to rise a year later, though it occurs less frequently than the previous terms. One explanation of this is that the Digital Twin is a subset, and has been developed in response to these prior terms. This is further investigated in Section 4 through co-occurrence analysis.

The remaining terms in Figure 5 have had relatively little uptake across academia to date. This could be due to the terms being in their infancy, or they could be the more granular terms that describe very specific concepts and therefore have reduced coverage. A third option is that these terms have been overshadowed by others or not proven to be successful in conveying a particular part of Digital Engineering. 


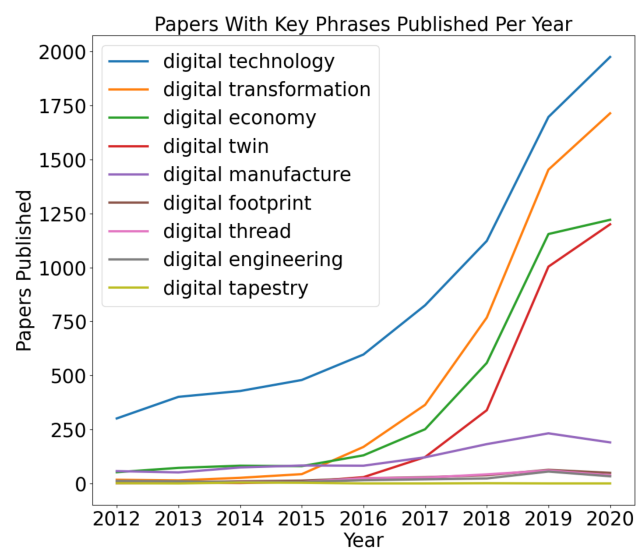

Figure 5. Number of documents with key terms published per year

\subsection{Digital ' $X$ ' Overlap - Co-Occurrence Analysis}

To investigate the overlap between the terms, the co-occurrence of each pair of terms is shown in Figure 6a. The values along the diagonal show the number of documents in which the term appears, and the values below the diagonal show the total number of documents featuring both terms in that pair, i.e., the overlap.

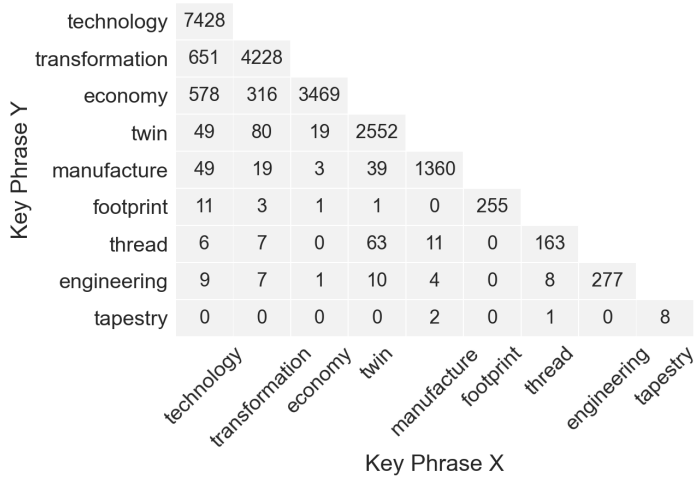

(a) Raw counts

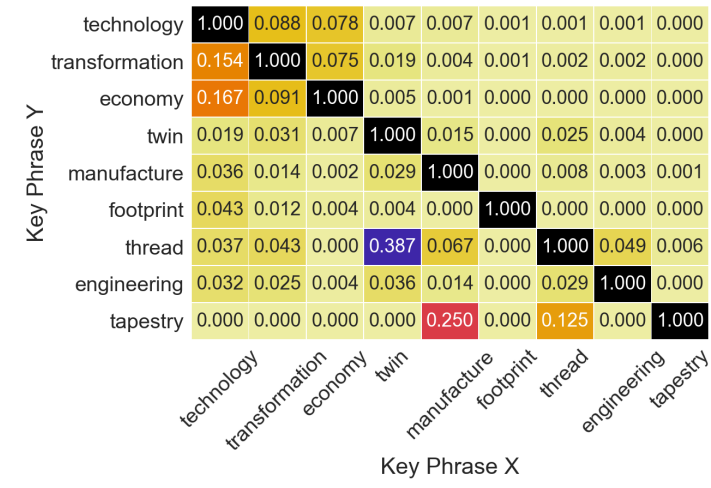

(b) Normalised co-occurrence

Figure 6. Co-occurrence of key phrases. [Note: The prevailing "digital" has been dropped for clarity]

Figure $6 \mathrm{~b}$ shows the normalised co-occurrence between each term pair as a heat-map. These values have been normalised with reference to both terms in the pair, with each cell showing the normalised impact of $t_{i}$ on $t_{j}$. For example, there are 163 documents containing "Digital Thread", 2,552 containing "Digital Twin" and the overlap is 63. Therefore the normalised impact of "Digital Twin" on "Digital Thread" is $63 / 163=0.387$, and the normalised impact of "Digital Thread" on "Digital Twin" is $63 / 2552=0.025$, as in Equation (1). The diagonal values show the impact of a term upon itself, so show a value of one. The co-occurrence of terms gives an insight into the existence of a relationship as well as its directionality. An equal normalised score between two terms indicates that both terms are of equal importance to each other. Digital Transformation, Economy and Technology all exhibit an equal relationship to one another, with values in the $0.075-0.175$ region demonstrating a significant overlap. This supports the idea raised in Section 3.3 that these three terms are all equally important, both to digital engineering and each other.

A highly directed relationship, like that of "Digital Twins" and "Digital Threads", indicates that one term may be a subset of the other. In this case, "Digital Twin" features in almost $40 \%$ of all documents concerning the "Digital Thread", whereas the "Digital Thread" only appears in $2.5 \%$ of documents containing "Digital Twin". This shows that the "Digital Thread" is likely to be a small part of the research into "Digital Twins", whereas a very large fraction of "Digital Thread" research is closely related to the "Digital Twin".

In Section 3.3 it was suggested that the Digital Twin is a subsidiary of the Digital Transformation, Economy and Technology terms. However the analysis in Figure 6 contradicts this statement, as there is minimal overlap between the Digital Twin and these terms. 
The remaining terms seem less strongly related. The terms "Digital Engineering" and "Digital Manufacture" seem to be loosely related to the "Digital Thread" and "Digital Twin" terms. However, Figure 5 shows that the term "Digital Manufacture' has had little increase in popularity over the last five years, implying that this term has not become fully associated with digital engineering and the remaining "Digital X" terms.

The term "Digital Footprint" is almost completely unrelated to any of the other terms, yet is still mentioned in over 250 documents. This is most likely due to the usage and definition of the term, as it is mostly used to describe the traceable online actions of an individual person rather than at an industrial scale.

\section{THE EMERGENT DIGITAL 'X' FRAMEWORK}

This section proposes a framework for "Digital X" terminology based on the emergent relationships seen in the literature. Evidence for strong links will be discussed and gaps in the knowledge will be highlighted.

\subsection{The Top of the "Digital X" Hierarchy}

As discussed in Section 3, Digital Technology, Transformation and Economy are the most successful terms. If one were to form a hierarchy and structure within Digital " $X$ ", it is argued that these would form the top level, as they were the first to start ramping up and are the most used terms. As these terms have been argued to be highly dependant on each other, it is suggested that these three terms are of equal importance and would tie for the top rank in the hierarchy.

\subsection{A Hierarchy Missing a Mid-Level}

Having identified the top level of the "Digital X" hierarchy, it is important to then map the space below. As discussed in Section 3, the relationship between the Digital Twin and the Digital Transformation, Technology and Economy is conflicted, with a strong temporal relationship but with minimal co-occurrence between terms. A possible explanation for this would be the existence of one or more intermediate terms that sit between them. By further analysing the co-occurence matrix in Figure $6 \mathrm{~b}$ we see that there is minimal overlap between the top three terms, and any other term in this dataset. This implies that any intermediate terms will be either a different "Digital X" term that was not identified or a term of different form, not investigated here. This opens up a new line of inquiry that should be explored that concerns the relationship between the "Digital X" terminology and related terms of different form.

\subsection{Twins and Threads}

Section 3.4 revealed an unbalanced relationship between the "Digital Thread" and the "Digital Twin" and it could be argued from this that the Digital Thread is a subset to the "Digital Twin". When looking at the temporal analysis we see that the "Digital Thread" has very few documents compared to the Digital Twin, but the publication rate in the last couple of years has started to rise. This could be the start of an exponential increase, similar to "Digital Twin" or "Digital Transformation" in previous years. This would be explained if the "Digital Thread" has been identified as a key area of research that could enable the Digital Twin, and is only at the start of its development.

\subsection{Unsuccessful Terminology}

Some of the search terms used have had limited uptake, such as "Digital Tapestry". This term was devised by Lockheed Martin SSC, who also coined the term "Digital Thread". The two terms were originally deeply linked, but "Digital Threads" have had significantly more success than "Digital Tapestries" to date. This could be explained by the broad set of definitions for the Digital Thread, some of which encapsulates the definition of Digital Tapestry (Table 2). It is possible that as the definition of the Digital Thread is later refined and focused that a separate term for Digital Tapestry may once again be required.

\subsection{The Emerging "Digital X" Framework}

Based on the findings, this paper proposes the "Digital X" framework shown in Figure 7. Confident links have been made where a likely link based on the evidence presented in this paper exist, and tentative links have been used where no link has been evidenced but it is suspected there will be a connection. 


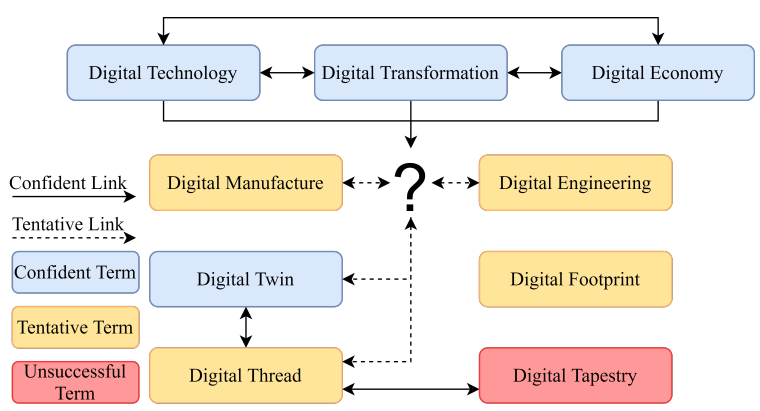

Figure 7. Proposed framework of "Digital X" terms

Terms have been ranked as confident, tentative or unsuccessful depending on whether or not they have shown a link to other terms in the system, whether they are in use within the space and whether they have a clear and consistent definition or use case. The terms "Digital Manufacture" and "Digital Engineering" do not have any strong links to other terms analysed in this paper, but it is expected that they are linked in some way to the rest of the framework. The "Digital Thread" is a distinct term, yet lacks a clear definition leaves it as a tentative term. The "Digital Footprint" is expected to be either distantly related or unrelated so no links have been assigned to it. The "Digital Tapestry" is known to have a link to the "Digital Thread" through Lockheed Martin, but has had negligible use outside of this company.

\subsection{A Cross-Check with Industry}

With the review having been centred around academic papers, it is important to perform a cross-check with accepted/emerging industry definitions to confirm alignment and consistency. To perform this check, the definitions used by Deloitte and Mckinsey \& Company ${ }^{2}$ have been collated and are shown in Table 4.

Most terms are similar across academic and industry domains, and this agreement indicates that this framework would be impactful for both. However, the industry definitions for Digital Threads are slightly contradictory, as Mckinsey appears to blur the lines between the Digital Thread and the Digital Twin. The two definitions of Digital Twins have differing levels of detail. This lack of consistency is also present in academia, indicating an area where further clarification would benefit both domains.

There is currently work in this space being conducted by groups such as ISO (e.g., ISO/TR 24464) and the Digital Twin Consortium ${ }^{3}$, though this work is by no means complete. Terms such as Digital Thread have had significantly less attention and as such are still very broad and inconsistent in definitions within and across fields. To this end, it is vital that all invested parties, including academia, industry, thought leaders and standards committees work together towards a shared understanding, ensuring that all definitions and use cases are consistent with each other.

\subsection{Implications for Engineering Design}

The work presented in this paper is only the first step towards fully defining the relationship between different aspects of Digital Engineering's Digital Landscape, and as the field matures, we expect it to have significant implications on Engineering Design research and practice:

1. By identifying the definitions and the relationships between these terms, future engineering design research and practice will be easier to articulate and associate with dimensions of digital engineering/digital transformation.

2. By highlighting the gaps and inconsistencies in understanding, further work, such as the need for standards, can be better targeted.

3. The lack of consistency in definitions of terms such as "Digital Thread" across related fields requires further work to bring together research, practitioners and professional bodies to resolve inconsistencies and generate the shared/common understanding.

4. A framework and lexicon is a prerequisite for industry to understand their current maturity and to determine how, where and when to deploy digital technologies in order to continue or start their Digital Transformation required to remain competitive. A common lexicon is also essential for devising and applying appropriate measures of performance.

\footnotetext{
${ }^{2}$ Two prominent engineering consultancy service providers.

${ }^{3}$ www.digitaltwinconsortium.org
} 
Table 4. Digital ' $X$ ' industry definitions.

\begin{tabular}{l|ll}
\hline$\#$ & Definition & Ref. \\
\hline Digital Transformation & \\
\hline 1 & $\begin{array}{l}\text { The use of technology to radically improve the performance or reach of an organisation } \\
\text { [Harnessing] the power of digital technology to rethink every aspect of the organisation }\end{array}$ & $\begin{array}{l}\text { (Deloitte, 2018a) } \\
\text { (Mckinsey, 2017) }\end{array}$ \\
\hline Digital Twin & \\
\hline 1 & $\begin{array}{l}\text { An evolving digital profile of the historical and current behaviour of a physical object } \\
\text { or process that helps optimise business performance. It is the exact digital replica of a } \\
\text { physical entity }\end{array}$ & (Deloitte, 2018b) \\
2 & $\begin{array}{l}\text { A digital representation of the physical asset } \\
\text { Digital Thread }\end{array}$ & (Mckinsey, 2021) \\
\hline 1 & $\begin{array}{l}\text { A single, seamless strand of data that stretches from the initial design concept to the fin- } \\
\text { ished part, constituting the information that enables the design, modelling, production, }\end{array}$ & (Deloitte, 2016) \\
& $\begin{array}{l}\text { use, and monitoring of an individual manufactured part } \\
\text { digital representation }\end{array}$ & (Mckinsey, 2015) \\
\hline 1 & $\begin{array}{l}\text { The economic activity that results from billions of everyday online connections among } \\
\text { people, businesses, devices, data, and processes }\end{array}$ & (Deloitte, 2019b) \\
\hline Digital Tapestry & $\begin{array}{l}\text { Using data from connected systems to create, innovate, and replicate components, prod- } \\
\text { ucts, and entire programs as part of a totally integrated system where data threads come } \\
\text { together, [bringing] people, processes, and tools into a common information framework } \\
\text { across the life cycle of its products }\end{array}$ & (Deloitte, 2019a) \\
\hline Digital Economy & \\
\hline 1 &
\end{tabular}

5. By formalising the relationship between different aspects of Digital Engineering, improvements in one area will be easier to relate to other areas, reducing duplication and enhancing cooperation between different stakeholders.

\section{CONCLUSION}

An increasingly convoluted lexicon is emerging to describe Digital Engineering's Landscape. As new technologies and systems are being developed by competing companies and academic bodies alike, the language used to describe this change is ungoverned and open to interpretation and significant confusion. Through analysis of the usage and temporal variation of key terms of the form "Digital X" combined with co-occurrence analysis across 19,627 documents, this paper has made the first important steps into the disambiguation and framing of this space. In this regard, an initial framework has been proposed that represents the interrelationships between the "Digital X" terms identified in this paper, though further work is required to fully map the space. Five expected implications on Engineering Design research and practice are proposed as a result of this, and further work; increasing the ease of articulation of Digital Engineering, allowing better targeting of valuable future work and actions, and enhancing cooperation between research, practitioners and professional bodies.

\section{ACKNOWLEDGEMENTS}

The work has been undertaken as part of the Engineering and Physical Sciences Research Council (EPSRC) grants - EP/R032696/1 and EP/V05113X/1 - and Digital Engineering and Technology \& Innovation (DETI) project. The author would like to thank the University of Bristol and Centre for Modelling and Simulation (CFMS) in supporting this research.

\section{REFERENCES}

Aghaei Chadegani, A., Salehi, H., Yunus, M., Farhadi, H., Fooladi, M., Farhadi, M., and Ale Ebrahim, N., 2013. A comparison between two main academic literature collections: web of science and scopus databases. Asian social science, 9(5), pp.18-26. 
Bullen, G.N., 2014. Digital manufacturing: the digital tapestry. (Technical report). SAE Technical Paper.

Callon, M., Courtial, J.-P., and Laville, F., 1991. Co-word analysis as a tool for describing the network of interactions between basic and technological research: the case of polymer chemsitry. Scientometrics, 22(1), pp.155-205.

Callon, M., Courtial, J.-P., Turner, W.A., and Bauin, S., 1983. From translations to problematic networks: an introduction to co-word analysis. Information (international social science council), 22(2), pp.191-235.

Deloitte, 2016. $3 d$ opportunity and the digital thread.

Deloitte, 2018a. Digital enablement, turning your transformation into a successful journey.

Deloitte, 2018b. Expecting digital twins.

Deloitte, 2019a. Aerospace \& defense 4.0, capturing the value of industry 4.0 technologies.

Deloitte, 2019b. What is digital economy?

DePaolo, C.A. and Wilkinson, K., 2014. Get your head into the clouds: using word clouds for analyzing qualitative assessment data. Techtrends, 58(3), pp.38-44.

Feeney, A.B., Frechette, S.P., and Srinivasan, V., 2015. A portrait of an iso step tolerancing standard as an enabler of smart manufacturing systems. Journal of computing and information science in engineering, 15(2).

Gainsburg, J., Rodriguez-Lluesma, C., and Bailey, D.E., 2010. A "knowledge profile" of an engineering occupation: temporal patterns in the use of engineering knowledge. Engineering studies, 2(3), pp.197-219.

Gaska, M.T., Bobinis, J.S., and Galluzzo, V., 2015. Application of system design for operational effectiveness for architectural modeling of the sos relationship between primary and enabling systems. Procedia computer science, 61, pp.240-245.

Girardin, F., Calabrese, F., Dal Fiore, F., Ratti, C., and Blat, J., 2008. Digital footprinting: uncovering tourists with user-generated content. Ieee pervasive computing, 7(4), pp.36-43.

GmbH, P.H. and Co., K., 1996. Digital thread counter - camscan 5200. German; English. Melliand textilberichte, 77(9), 570-571+E116.

Gopsill, J., Humphrey, M., Thompson, D., and Garcia, E., 2020. Co-word graphs for design and manufacture knowledge mapping. Proceedings of the design society: design conference, 1, pp.1275-1284.

Hess, T., Matt, C., Benlian, A., and Wiesböck, F., 2016. Options for formulating a digital transformation strategy. Mis quarterly executive, 15(2).

Kim, D.B., Witherell, P., Lipman, R., and Feng, S.C., 2015. Streamlining the additive manufacturing digital spectrum: a systems approach. Additive manufacturing, 5, pp.20-30.

Kinberg, C. and Landeck, B., 1970. Integrated manufacturing systems. architectural considerations. Ibm journal of research and development, 14(6), pp.589-604.

Loper, E. and Bird, S., 2002. Nltk: the natural language toolkit. In proceedings of the acl workshop on effective tools and methodologies for teaching natural language processing and computational linguistics. philadelphia: association for computational linguistics.

Matt, C., Hess, T., and Benlian, A., 2015. Digital transformation strategies. Business \& information systems engineering, 57(5), pp.339-343.

Mckinsey, 2015. Industry 4.0, how to navigate digitization of the manufacturing sector.

Mckinsey, 2017. A roadmap for a digital transformation.

Mckinsey, 2021. Road work ahead: the emerging revolution in the road construction industry.

Mies, D., Marsden, W., and Warde, S., 2016. Overview of additive manufacturing informatics:“a digital thread”. Integrating materials and manufacturing innovation, 5(1), pp.114-142.

O'Keeffe, G.S., Clarke-Pearson, K., Communications, C. on, and Media, 2011. The impact of social media on children, adolescents, and families. Pediatrics, 127(4), pp.800-804.

Rosen, R., Von Wichert, G., Lo, G., and Bettenhausen, K.D., 2015. About the importance of autonomy and digital twins for the future of manufacturing. Ifac-papersonline, 48(3), pp.567-572.

Steuben, J.C., Iliopoulos, A.P., and Michopoulos, J.G., 2016. Implicit slicing for functionally tailored additive manufacturing. Computer-aided design, 77, pp.107-119.

Stuehler, J., 1970. Integrated manufacturing process control system. implementation in ibm manufacturing. Ibm journal of research and development, 14(6), pp.605-613.

Sturm, L.D., Williams, C.B., Camelio, J.A., White, J., and Parker, R., 2017. Cyber-physical vulnerabilities in additive manufacturing systems: a case study attack on the. stl file with human subjects. Journal of manufacturing systems, 44, pp.154-164.

Tao, F., Cheng, J., Qi, Q., Zhang, M., Zhang, H., and Sui, F., 2018. Digital twin-driven product design, manufacturing and service with big data. The international journal of advanced manufacturing technology, pp.3563-3576.

Yang, X., Dong, A., and Helander, M., 2012. The analysis of knowledge integration in collaborative engineering teams. Journal of engineering design, 23(2), pp.119-133. 